\title{
Excessive proinflammatory cytokine and chemokine responses of human monocyte-derived macrophages to enterovirus 71 infection
}

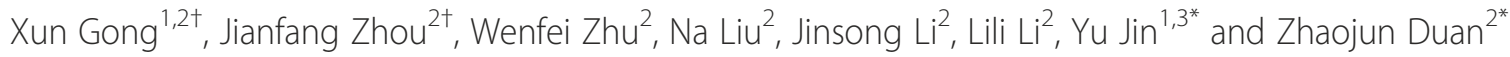

\begin{abstract}
Background: The levels of proinflammatory cytokine or chemokine in blood and cerebrospinal fluid are thought to be one of predictors for clinical severity of enterovirus 71 (EV71) infection, yet the cellular sources or signalling mechanism remain undefined. Here, we focused on the response of human primary monocyte-derived macrophages (MDMs) to EV71 virus and its possible mechanisms.

Methods: Human primary MDMs were infected by EV71 virus in vitro. Infectivity and viral replication were assayed, and cytokine responses were determined by Cytometric Bead Array(CBA) analysis. The relative changes of Toll-like receptors, retinoic acid-inducible gene I (RIG-I) and melamoma differentiation associated gene 5 (MDA5) mRNA expression were detected by real-time RT-PCR.

Results: Effective infection and viral replication were detected in EV71-infected MDMs. The titters of progeny virus released from EV71-infected MDMs gradually increased from 6-h to 48-h point of infection (POI.). Proinflammatory cytokines: IL-1, IL-6, TNF-a but not IFN-a and $\gamma$ were induced in MDMs by EV71. EV71 infection significantly increased the release of IL-8, IP-10 and RANTES at 12-h or 24-h POI.. Upregulation of TLR2, TLR7 and TLR8 mRNA expression rather than TLR3, TLR4, TLR6, TLR9, TLR10, RIG-I, MDA5 were found at different time points in EV71-infected MDMs.

Conclusions: Our findings suggested that macrophages are not only the important target cells but also the effectors during EV71 infection, and they may play an important role in the pathogenesis of EV71 infection. And the proinflammatory cytokine and chemokine responses in EV71-infected MDMs may be mediated by the activation of differential pattern of TLRs.
\end{abstract}

Keywords: Enterovirus 71, Macrophages, Proinflammatory cytokines, Chemokines, Toll-like receptors

\section{Background}

Enterovirus 71, a positive-stranded RNA virus, is highly infectious and can cause hand-foot-and-mouth disease (HFMD), herpangina, neurological diseases with potentially more serious complications such as encephalitis, aseptic meningitis, brain stem encephalitis (BE) in infants and young children. Many of patients died from fulminant pulmonary edema (PE) or hemorrhage, which

\footnotetext{
* Correspondence: jinyuldyy@163.com; zhaojund@126.com

${ }^{\dagger}$ Equal contributors

'School of Basic Medical Sciences, Lanzhou University, Lanzhou 730000, PR China

${ }^{2}$ National Institute for Viral Disease Control and Prevention, China CDC, Beijing 100052, PR China

Full list of author information is available at the end of the article
}

was based on nervous system injury [1,2]. In recent years, its prevalence in Malaysia, Taiwan, Singapore, China, Korea and so on, and continuous spreading widely provoked global concern [3-7].

Although the pathogenesis of neurogenic PE caused by EV71 remains unclear, host factors especially host immune response rather than EV71 itself or its genotype may be one of important determinants for the disease severity $[1,8]$. Excessive proinflammatory cytokine and chemokine responses were thought to contribute to the severity of EV71 infection [9]. Current findings implied that the inflammatory cytokines or chemokines are probably synthesized by infiltrated mononuclear cells (macrophages or $\mathrm{T}$ cells) in tissues or neuron-surrounding cells, such as microglia (the resident macrophages in

\section{Biomed Central}


central nervous system (CNS)) or astrocytes in EV71 infection [9-11]. Since monocyte-derived macrophages and microglia are derived from the common precursors in the bone marrow, express similar surface markers and perform roughly similar functions [12], both of them may be involved in the immune response to EV71 infection.

Previous studies have identified that the immune cells such as human peripheral blood mononuclear cells, human T cell line (Jurkat), monocytic cells, human immature dendritic cells can be infected with EV71 [13-15]. Macrophages play an important role in the innate immunity system, however, the interaction between macrophages and EV71 remains unknown. Furthermore, Toll-like receptors (TLRs) and RIG-I-like helicases (RLHs) recognize a number of viruses resulting in the activation of an innate immunity response that induce secretion of proinflammatory cytokines and chemokines [16-19]. In this study, we focused on the proinflammatory cytokine and chemokine responses of MDMs to EV71 infection. And we also investigated whether TLRs and RLHs were involved in EV71-infected MDMs, and explored the possible mechanisms of inflammatory responses to EV71 infection.

\section{Results}

Effective infection and viral replication of EV71 in human MDMs

We first determined the infectivity of MDMs to EV71 by immunofluorescence staining of viral VP1. As evidenced by the expression of viral $\mathrm{VP}^{+}$at 12 -h POI. (data not shown), human MDMs could be infected by EV71 virus.
Furthermore, the infection showed a dose-dependent for infectious dose, as evidenced by an increase of VP1 expression at 24-h POI. along with a MOI of 0.1, 0.5, 1 and 5. (Figure 1C-F). The VP1+ fluorescence signal was detected in the cytoplasm in EV71-infected MDMs at 24-h POI., but not in mock or UV-inactivated EV71-infected MDMs at MOI of 5(Figure 1). Then the viral gene copy was quantified. An increase of viral gene copy was detected between 6-h and 12-h POI. (range from 16,421 \pm 5061 to $136,027 \pm 54,473$ copies $/ 10^{4}$ copies of beta-actin, $p=0.008$, $\mathrm{n}=8$ ). The viral gene copies maintained at a high level at the following assessed time points, 24-h and 48-h POI, but showed no significant difference from that at 12-h POI. $(p=0.194, p=0.273, \mathrm{n}=8)$ (Figure 2A). To identify whether MDMs produce infectious progeny particles or not, virus titers of culture supernatants were performed by measuring the $50 \%$ tissue culture infective dose (TCID50) on Vero cells and calculated by using the Reed and Muench formula [20]. The results showed that MDMs were productively infected by EV71 with approximate 10-fold increase in virus titter by 48 -h POI. (Figure $2 \mathrm{~B}, \mathrm{n}=8$ ). Our in vitro findings suggested that MDMs are susceptible to EV71 infection and maybe one of target cells in vivo during EV71 infection.

\section{Enhanced proinflammatory cytokine responses of human MDMs to EV71}

We subsequently determine the functional response of human MDMs to live EV71 or UV-irradiated EV71. Significant higher level of TNF- $\alpha$ was rapidly induced by live
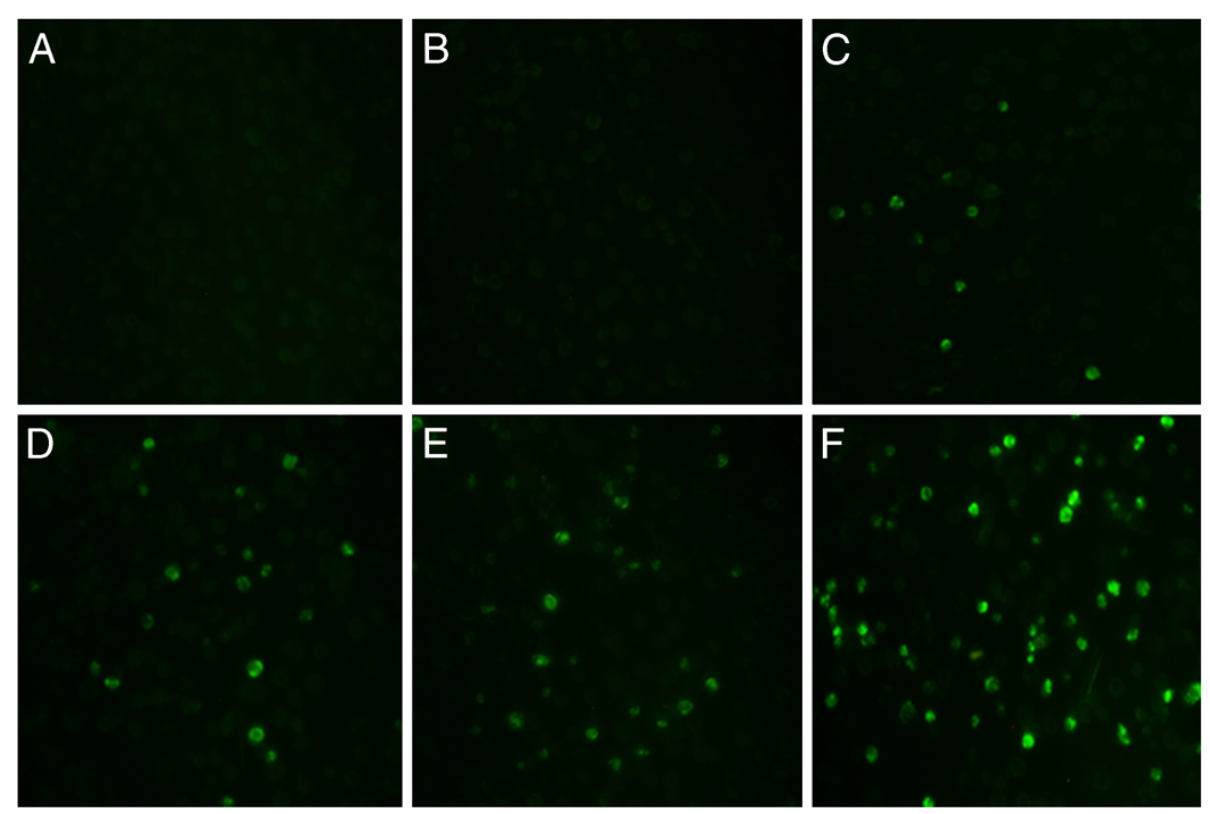

Figure $1 \mathrm{~A}$ dose-dependent infection of EV71 virus in human monocyte-derived macrophages (MDMs). Immunofluorescence staining of EV71 VP1 in mock- or EV71-infected MDMs was assayed at 24-h POI.. A. mock, B. UV-inactivated EV71 infection, C. EV71 infection at a MOI of 0.1, D. EV71 infection at a MOI of 0.5, E. EV71 infection at a MOI of 1, F. EV71 infection at a MOI of 5. 


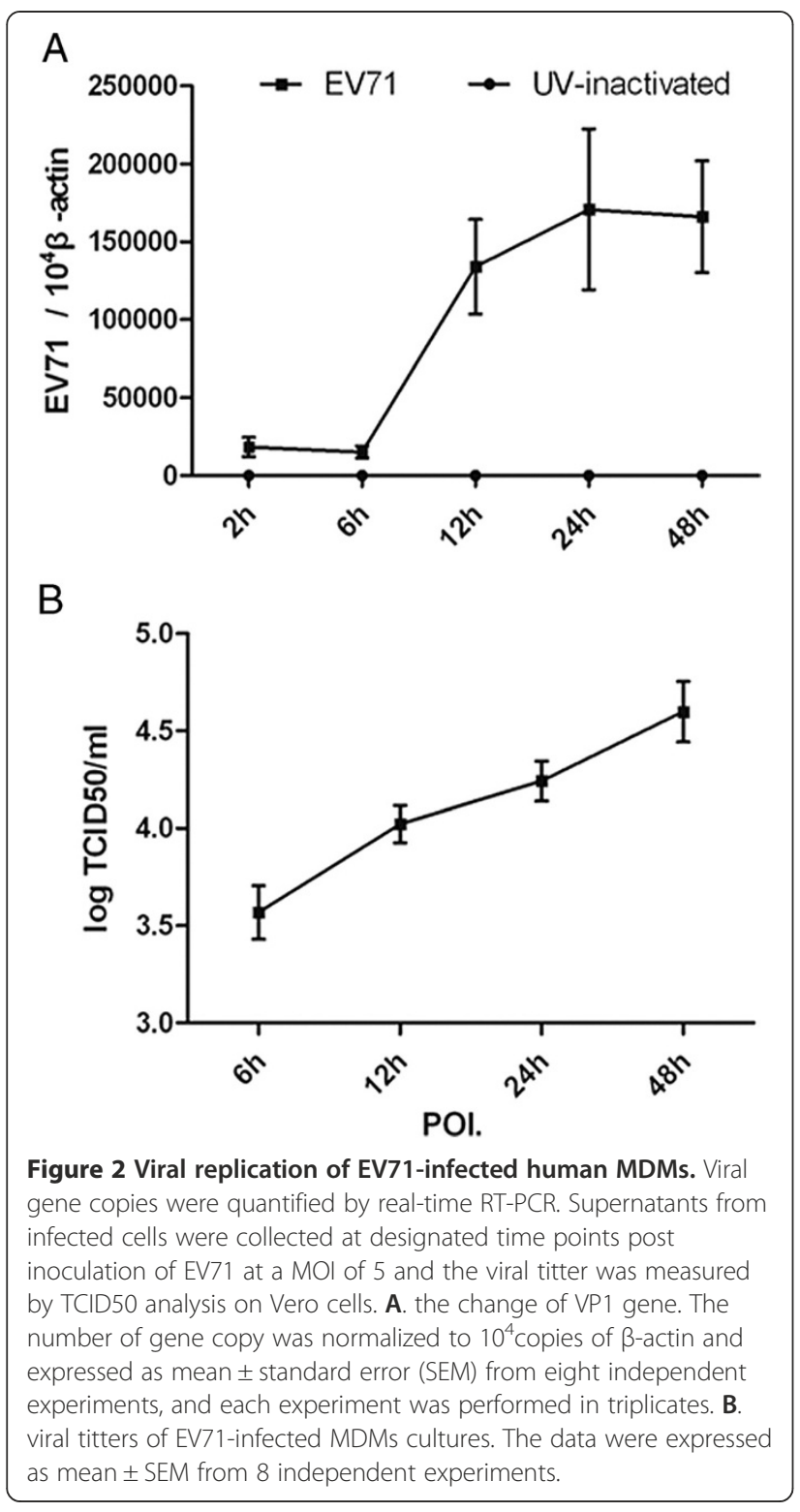

EV71 infection other than mock or UV-irradiated EV71 at the 12-h POI. $(72.34 \pm 52.56 \mathrm{pg} / \mathrm{ml}$ vs. $6.68 \pm 7.67 \mathrm{pg} / \mathrm{ml}$, $31.74 \pm 20.26 \mathrm{pg} / \mathrm{ml}$, respectively, $p=0.001, p=0.011$, $\mathrm{n}=8$ ), and then gradually decreased over time $(30.68 \pm 21.50 \mathrm{pg} / \mathrm{ml}$, at $24-\mathrm{h}$ POI.). Both IL-1 $\beta$ and IL-6 from MDMs were triggered by live EV71, and maintained a higher level at 12-h $(p=0.018, p=0.008, \mathrm{n}=8)$ and 24-h POI. $(p=0.007, p=0.006, \mathrm{n}=8)$ as compared with that of mock (Figure 3). In addition, we found that EV71 infection failed to induce IFN- $\alpha$ releasing from human MDMs, and the IFN- $\gamma$ was undetectable.

\section{Enhanced production of IL-8, RANTES and IP-10 from human MDMs infected with EV71}

Chemokines include a large superfamily and play multiple roles in shaping the innate and adaptive immune responses during viral infection. We measured the levels of the chemokines including CXCL-10/IP-10, CCL-2/MCP-1, CXCL-9/MIG, CXCL-8/IL-8, and CCL-5/RANTES in the supernatants at $12-\mathrm{h}$ and 24 -h POI. At early time points (12-h POI.), enhanced production of IL-8 and RANTES was detected in EV71-infected MDMs than in mockinfected MDMs $(p=0.003, p=0.002, \mathrm{n}=8)$, and the concentrations of these chemokines increased along with time (Figure 4). At 24-h POI, much higher concentrations of IL-8, RANTES and IP-10 were found in EV71-infected MDMs than in those of $\operatorname{mock}(p=0.013, p=0.004$, $p=0.001, \mathrm{n}=8)$. Moreover, compared with mock-infected MDMs, UV-irradiated EV71 can induce significantly high level of IL-8 production at 12-h and 24-h POI. than mock $(p=0.004, p=0.002, \mathrm{n}=8)$.

\section{Up-regulation of TLR2, TLR7 and TLR8 mRNA expression in EV71 infected- MDMs}

In order to investigate the involvement of TLRs and RLHs in EV71-infected MDMs, we screened the TLR2, TLR3, TLR4, TLR6, TLR7, TLR8, TLR9, TLR10, RIG-1, Mda-5 mRNA expression by real-time quantitative RT-PCR. The results showed that the mRNA expression of TLR2 appeared to be significantly enhanced in EV71-infected human MDMs from 6-h to 24-h POI.. Increased mRNA expressions of TLR2 were also observed in UV-inactivated virus-infected MDMs at 12 -h to 24 -h POI. as compared with mock $(p=0.014, p=0.008, \mathrm{n}=8)$. Both TLR7 and TLR8 mRNA expressions were significantly enhanced in EV71-infected MDMs at different time points. In contrast to the findings that higher TLR7 mRNA level was induced at earlier time points (6-h and 12-h POI.), enhanced mRNA expression of TLR8 was observed at a relatively later stage, 24-h POI. (Figure 5). In addition, there was minor change in the mRNA expression of TLR3, TLR4, TLR6, TLR9, TLR10, RIG-1 and Mda- 5 in the tested samples.

\section{Discussion}

Macrophages, which are shown to support the infection of various viruses including HIV-1, influenza viruses, and poliovirus and so on, play a critical role in presentation of antigens, pathogen clearance, and induction of inflammation during the early phase of viral infection [21-23]. In this study, both viral gene and antigen of EV71 were detected. The increases of virus yields and the number of viral gene copies were observed in EV71-infected MDMs between the 6-h and 48-h POI.. Excess cytokine and chemokine responses of MDMs were triggered by EV71. These findings suggested that macrophages may be not only the target cells but also the effectors during EV71 infection.

It is controversial whether TNF- $\alpha$ was involved in fatal EV71 infection. Significant or minor change of blood TNF- $\alpha$ in EV71 patient with both encephalitis and PE was reported by different clinical studies [24,25]. 

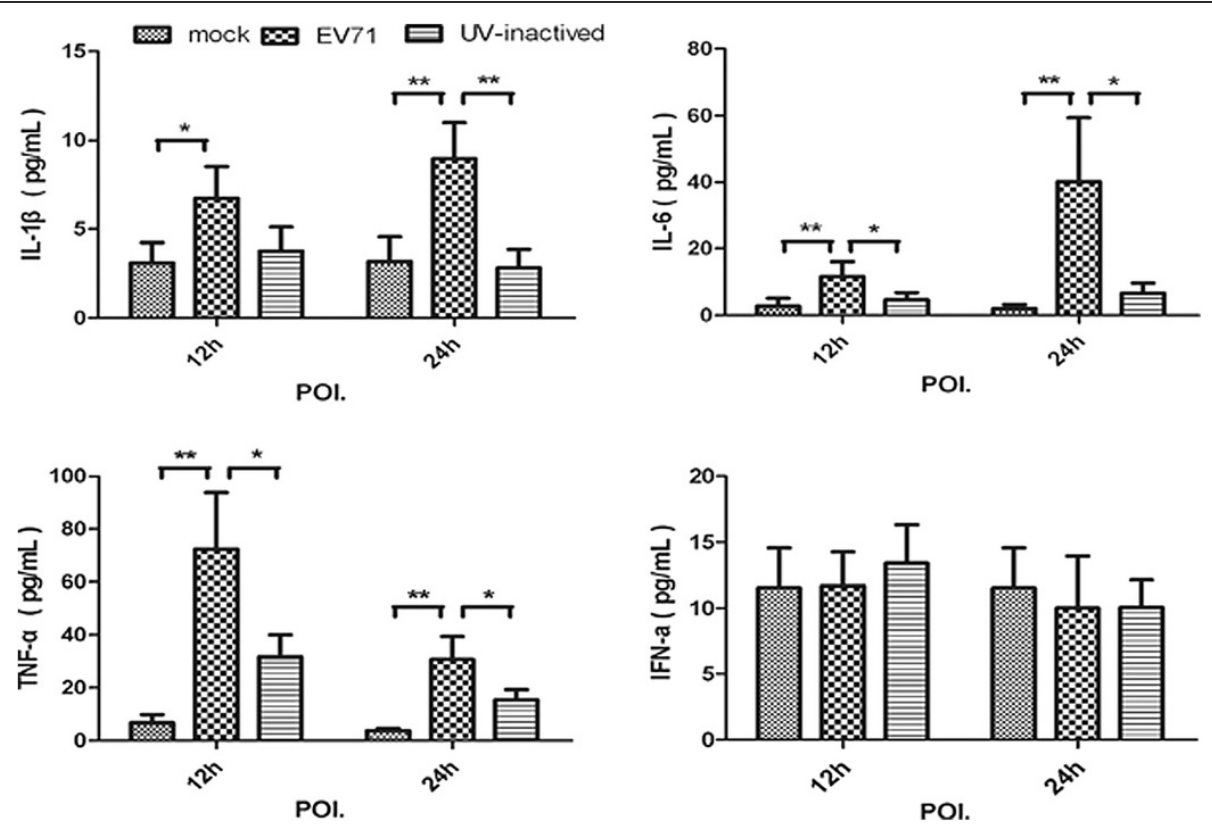

Figure 3 Proinflammatory cytokines IL-1 $\beta$, IL- 6 and TNF- $\alpha$ induced in EV71 infected MDMs. MDMs were infected with or without EV71 (live or UV-inactivated) at MOI of 5. Culture supernatants were harvested at 12-h and 24-h after infection to measure the indicated cytokines by CBA as described in Methods section. Data are expressed as mean \pm SEM from eight independent experiments performed in triplicates. $\left({ }^{*}, P<0.05\right.$ and $\left.{ }^{*}, P<0.01\right)$.
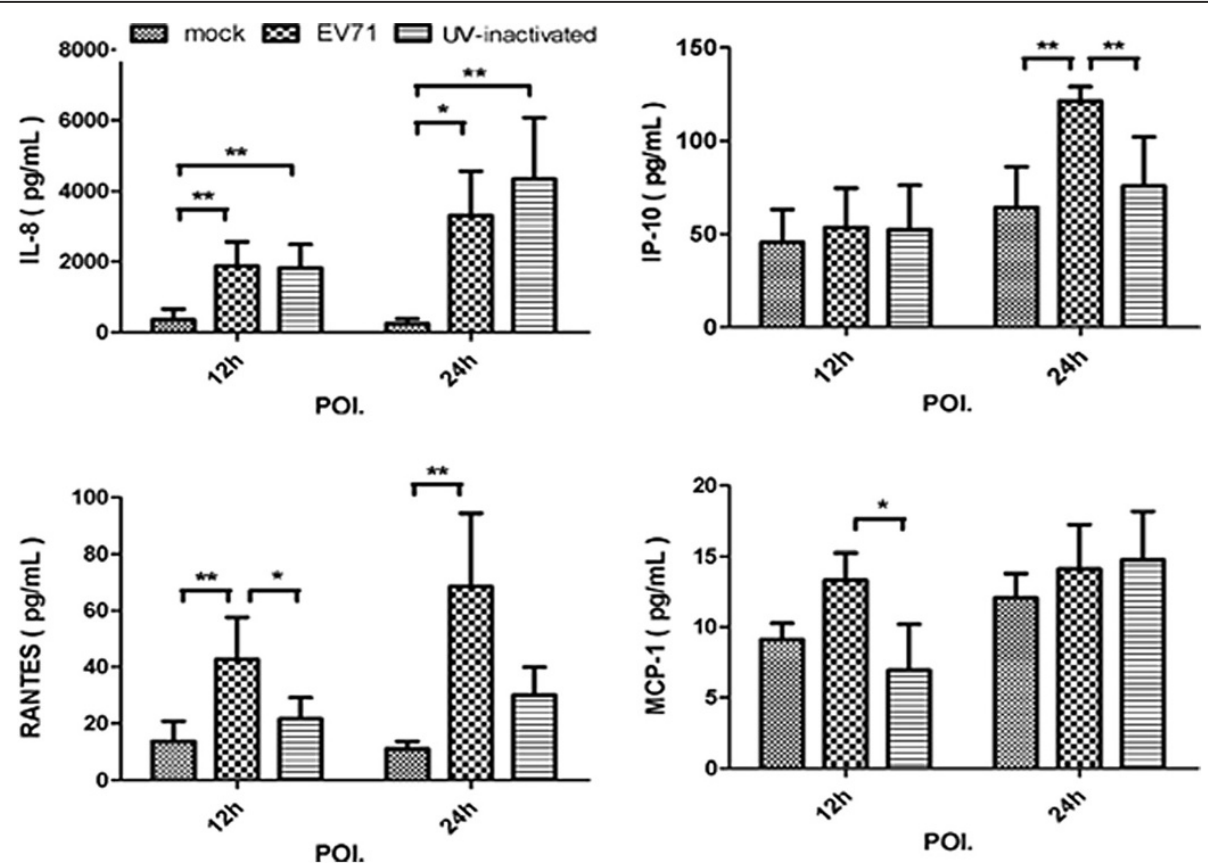

Figure 4 Chemokines induced in MDMs infected with EV71 and (or) UV-inactivated EV71. MDMs were infected with or without EV71 (live or UV-inactivated) at MOI of 5 for 12-h and 24-h. The chemokines including IP-10, MCP-1, RANTES, IL-8, MIG concentration in the supernatants at $12-\mathrm{h}$ and $24-\mathrm{h}$ was measured by CBA. Data are expressed as mean \pm SEM from eight independent experiments performed in triplicates. ${ }^{*}, \mathrm{P}<0.05$ and $\left.{ }^{* *}, \mathrm{P}<0.01\right)$. 

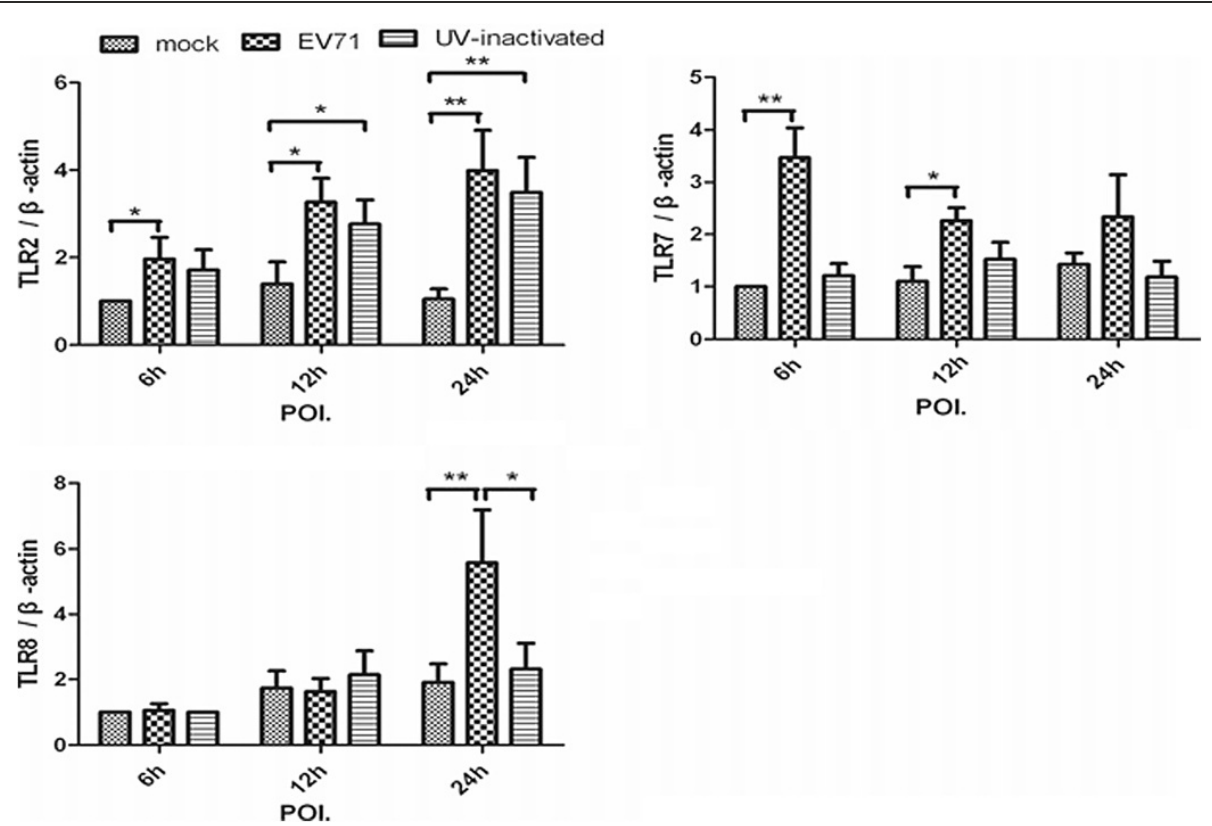

Figure 5 A higher mRNA expression of TLR2, TLR7 and TLR8 were induced in MDMs infection with EV71. The mRNA expression of TLR2, TLR3, TLR4, TLR6, TLR7, TLR8, TLR9, TLR10, RIG-1 and MDA-5 were performed by SYBR green real-time RT-PCR. The relative change of mRNA expression was analyzed using $\Delta \Delta C T$ method. To standardize results for variability in CDNA quantity, we expressed them with target gene/ $\beta$-actin as 1 in mock at 6-h POI.. Data are expressed as mean \pm SEM from eight independent experiments performed in triplicates $\left({ }^{*}, P<0.05\right.$ and $\left.{ }^{*}, P<0.01\right)$.

Significant stronger release of TNF- $\alpha$ from EV71-infected MDMs at 12-h POI than 24-h POI in the study, implying that TNF- $\alpha$ was induced by EV71 infection at early stage and maybe involved in its pathology. Consistent with clinical features of EV71 patients with encephalitis and PE, who are presented with higher levels of the proinflammatory cytokines in blood $[9,24]$, IL-6, IL- $1 \beta$ and TNF- $\alpha$ were induced in EV71-infected MDMs. These proinflammatory cytokines are thought to be the potent pyrogens inducing fever, and the magnitude of febrile response correlates with the level of virus shedding in human and animals [26]. Notably, a transient increase of blood brain barrier (BBB) permeability and its injury were found at early stage of EV71 infection [27]. The pathology may be due to an augmentation of systemic and local TNF- $\alpha$ production, which exhibits detrimental effects by enhancing cell infiltration, cytopathic damage, or functioning as a paracellular pathway for the virus across the BBB [28]. Furthermore, the subsequent responses of acute phase proteins and chemokine activations mediated by IL-6, IL- $1 \beta$ and TNF- $\alpha$ could exacerbate virus induced inflammation and pathology [29]. Therefore, the rapid and strong proinflammatory response of MDMs to EV71 may partially explain the clinical severity.

Macrophages or plasmacytoid DCs, specialized in secreting large amounts of type I IFN after virus infection, play an important role in viral infection. However, minor change of IFN- $\alpha$ in EV71-infected MDMs was detected in our study. It is likely that the $3 \mathrm{C}$ protein of EV71 virus inhibits type I interferon activation by viral nuclear acid or RIG-I signalling [30]. Although elevated level of IFN- $\gamma$ in both plasma and cerebrospinal fluid was found in patients with PE [10], the IFN- $\gamma$ from EV71-infected MDMs was undetectable here. The discrepancy may be a result of the main cellular source of IFN- $\gamma$ in vivo from activated $\mathrm{T}$ and NK cells other than macrophages. A series of IFN- $\gamma$-responsive and inflammatory chemokines such as RANTES, IP-10 and IL-8 in MDMs were triggered by EV71 virus. Not only live- but also UV-inactivated EV71 can induce IL-8 releasing from MDMs, which suggested that viral proteins may also be involved in inducing of IL-8. IL-8 is a potent chemoattractant and activator of neutrophils, one of the major immune cells responsible for inflammation of CNS during meningitis or encephalitis [31]. Our in vitro findings support the clinical findings that a higher total WBC count, absolute neutrophil count and elevated IL-8 and IP-10 level in patients with BE or PE [11,25].

TLRs and RLHs recognize distinct ligands and trigger host immune response in different virus infection [32]. The recognition of human rhinovirus, human parechovirus 1 , rotavirus or coxsackie B virus by different host cells are mediated through elevated TLR2, 7, 8 and (or) Mda5 expression, which induce secretion of proinflammatory cytokines, chemokines, and interferons [16-19]. When TLR2, TLR7 and TLR8 were silenced, there was a considerable decrease in cytokine secretion in human 
airway epithelial cells with HRV-6 infection [18]. In our study, elevated TLR2, TLR7 and TLR8 expressions as well as increased proinflammatory cytokines and chemokines were observed in EV71-infected MDMs. Furthermore, enhanced IL-8 and TLR2 mRNA expression were also found in UV-inactivated virus treated-MDMs. It is likely that the interaction between TLR2 on cell surface and viral proteins rather than viral RNAs is necessary for the activation of MDMs. Significant up-regulations of mRNA for TLR7 and TLR8 were observed at different time points, and it suggested that there were differential kinetics between TLR7 and TLR8 involvements in EV71-infected MDMs. These results indicated the cytokine productions in EV71-infected MDMs may be partly through the activation of TLR2, TLR7 and (or) TLR8. Further investigations such as gene knockout experiments are needed to determine the exact roles of them.

As documented previously, younger children less than 5-year-old were the most susceptible groups to EV71 and usually presented with severe infection [3]. The underlying mechanisms for the severity remain unknown. And enhanced proinflammatory cytokines and chemokines were indeed found in children patients with encephalitis or PE $[9,11,24]$. Although there is differential expression level of chemokine receptors on adult and neonatal MDMs [33], one of possible factors for the age-related severity in avian influenza virus infection, a similar cytokines/chemokines profile was found in influenza virus-infected adult and neonatal MDMs and the levels of most of the cytokines/chemokines were comparable [23]. Moreover, the adults can also be infected with EV71 and the clinical severity in adult patients with acute encephalitis was similar to those of EV71 infection in children [34]. Therefore, the findings on adult MDMs infection model here may partially reflect natural in vivo infection.

\section{Conclusions}

In summary, effective viral replication in EV71-infected MDMs and excessive inflammatory cytokine and chemokine responses of MDMs to the virus were demonstrated for the first time in our study. The results indicate that macrophages are an important target for EV71, and they can trigger pro-inflammatory response and chemokine response against viral infection. However, inordinate macrophages response may be detrimental to the infected host due to exacerbate virus inflammation and pathology. TLR2, TLR7 and TLR8 may participate in the induction of cytokines/chemokines in EV71-infected MDMs. These data suggested that MDMs may play an important role in the pathogenesis of EV71 infection in vivo though more evidence is needed.

\section{Methods}

Isolation and culture of monocyte-derived macrophages

The study was approved for human subject protection by the Ethics Committee of National Institute for Viral Disease Control and Prevention, China CDC. Following informed consent was written by participants.

Peripheral blood obtained from 8 healthy blood donors aged from 20 to 40 years old. Peripheral blood mononuclear cells (PBMCs) were isolated by a Ficoll-Paque density gradient (Pharmacia Biotech, Uppsala, Sweden) to remove erythrocytes, platelets, and cell debris. Monocytes were isolated by plastic adherence, harvested, counted and seeded on tissue culture plates in RPMI 1640 (Invitrogen Life Technologies, Great Island, NY, USA) medium supplemented with $10 \%$ heat-inactivated autologous plasma at $10^{6} \mathrm{cells} / \mathrm{ml}$. The purity of monocytes was determined by flow cytometry with anti-CD14 monoclonal antibody (Mab, PharMingen, San Diego, CA, USA) and was consistently above $90 \%$. The monocytes were reseed with $2-5 \times 10^{5}$ cells per well onto a 24 well culture plate, and were allowed to differentiate into MDMs for 10-14 days in vitro.

\section{Infection of MDMs with EV71}

Enterovirus 71 virus was propagated in RD cells (obtained from ATCC) in DMEM containing 2\% fetal bovine serum (FBS, Invitrogen, Grand Island, NY, USA) and incubated at $35^{\circ} \mathrm{C}$ with $5 \% \mathrm{CO}_{2}$. When $80 \%$ of the cells showed the typical enteroviral cytopathic effect (CPE), the infected cells were harvested after being frozen and thawed for three times. Cell debris was removed by centrifugation and filtration using a $0.22 \mu \mathrm{m}$ membrane filter (Millipore, Billerica, CA). EV71 viruses were inactivated using ultraviolet radiation $3000 \mathrm{mj} / \mathrm{cm}^{2}$, 30 mins on ice (UV-inactivated EV71). Differentiated MDMs were infected by EV71 and UV-inactivated EV71 at MOI (multiplicity of infection) of $0.1,0.5,1$ and 5 . This is taken to be 0 -h point of infection (POI.) for the experiments described below. Virus titters were performed by measuring the $50 \%$ tissue culture infective dose (TCID50) on Vero cells and calculated by using the Reed and Muench formula.

\section{Immunofluorescence assay of viral VP1 protein in infected cells}

MDMs were fixed with methanol: acetone (1:1) for $5 \mathrm{~min}$. The cell monolayer was incubated by anti-EV71 VP1 monoclonal antibody (MAB979, Millipore, Billerica, $\mathrm{CA}$ ) at room temperature for $60 \mathrm{~min}$, and followed by labelling FITC-conjugated goat anti-mouse IgG for 1-h. After being completely washed, the cells were observed under a fluorescence microscope. 
Table 1 Primer sequences and probes used in real-time PCR assay

\begin{tabular}{|c|c|c|c|}
\hline Gene & Primer and probe sequence (5/to3/) & Product length(bp) & GenBank \\
\hline \multirow[t]{3}{*}{ EV71 } & (R) CCCGCTCTGCTGAAGAAACT & 89 & AF302996 \\
\hline & $\overline{\text { (F)AGTGATGAGAGTATGATTGAGACACG }}$ & & \\
\hline & $\overline{\text { (P) TCGCACAGCACAGCTGAGACCACTC }}$ & & \\
\hline \multirow[t]{3}{*}{$\beta$-actin } & (R) CAAGTACTCCGTGTGGATCG & 90 & NM_001101.3 \\
\hline & (F) GGATGCAGAAGGAGATCACTG & & \\
\hline & (P) CCCTGGCACCCAGCACAATGA & & \\
\hline \multirow[t]{2}{*}{ RIG-1 } & (R) CCTCTGCACTGTTGCTCAGGAC & 192 & NM_004585.3 \\
\hline & (F) CTCTTGGCTTCGAGATGGCTTC & & \\
\hline \multirow[t]{2}{*}{ MDA5 } & (R) ATTGGTGACGAGACCATAACGGATA & 196 & NM_022168.2 \\
\hline & (F) AGGAGTCAAAGCCCACCATCTG & & \\
\hline \multirow[t]{2}{*}{ TLR2 } & (R) CACAAAGTATGTGGCATTGTCCAG & 158 & NM_003264.3 \\
\hline & (F) GTGTTGCAAGCAGGATCCAAAG & & \\
\hline \multirow[t]{2}{*}{ TLR3 } & (R) AGTGCCGTCTATTTGCCACACA & 181 & NM_003265.2 \\
\hline & (F) AACAGTGCACTTGGTGGTGGAG & & \\
\hline \multirow[t]{2}{*}{ TLR4 } & (R) ATGCGGACACACACACTTTCAAATA & 143 & NM_138554.3 \\
\hline & (F) TTGAGCAGGTCTAGGGTGATTGAAC & & \\
\hline \multirow[t]{2}{*}{ TLR6 } & (R) AGCCTTCAGCTTGTGGTACTTGTTG & 138 & NM_006068.2 \\
\hline & (F) CAGAGTGAGTGGTGCCATTACGA & & \\
\hline \multirow[t]{2}{*}{ TLR7 } & (R) TCTTCAACCAGACCTCTACATTCCA & 172 & NM_016562.3 \\
\hline & (F) GGAACATCCAGAGTGACATCACAG & & \\
\hline \multirow[t]{2}{*}{ TLR8 } & (R) GATTGCTGCACTCTGCAATAACTGA & 196 & NM_138636.3 \\
\hline & (F) GCTGCTGCAAGTTACGGAATGA & & \\
\hline \multirow[t]{2}{*}{ TLR9 } & (R) CAGGGCCTTCAGCTGGTTTC & 97 & NM_017442.2 \\
\hline & (F) CCGTGACAATTACCTGGCCTTC & & \\
\hline \multirow[t]{2}{*}{ TLR10 } & (R) TGTGTTGCAAGATAATTCGTGGAGA & 103 & NM_001017388.1 \\
\hline & (F) CATGATGGTTGGATGGTCAGATTC & & \\
\hline \multirow[t]{2}{*}{$\beta$-actin } & (R) CTAAGTCATAGTCCGCCTAGAAGCA & 186 & NM_001101.3 \\
\hline & (F) TGGCACCCAGCACAATGAA & & \\
\hline
\end{tabular}

\section{Quantification of mRNA by real-time RT-PCR}

Infected MDMs cultured in macrophage serum-free medium (Invitrogen, Grand Island, NY, USA) were harvested at 2-h, 6-h, 12-h, 24-h and 48-h POI. Total RNAs were extracted using QIAGEN RNeasy mini kit (QIAGEN, Hilden, Germany). Reverse transcription was performed on DNase-treated total RNA. The cDNA was synthesized from mRNA with oligo(dT) ${ }_{12-18}$ primer and Superscript II reverse transcriptase (Invitrogen, Grand Island, NY, USA). Specific primers and probes used in the real-time PCR assay were listed in Table 1 and Q-PCR were performed by Rote-Gene 3000 Sequence Detection System (QIAGEN, Hilden, Germany). Viral gene copies were quantified on the basis of a TaqMan Probes fluorescence signal after PCR. We expressed viral gene variability as the number of target gene copies per $10^{4}$ copies of $\beta$-actin. The relative changes of other human genes were analyzed by SYBR green real-time PCR. Dissociation curve analysis was performed after each assay, to ensure specific target detection.

\section{Measurement of cytokines}

Concentration of cytokines from culture supernatants were determined by Cytometric Bead Array(CBA). IL-1 $\beta$, IL-6, TNF- $\alpha$, IFN- $\alpha$, IFN- $\gamma$, IP-10, IL-8, RANTES, MCP-1, MIG Flex Set reagents (BD Biosciences, San Diego, CA) were used to measure cytokines by flow cytometry according to the manufacturer's protocol. The results are presented as the means of assays performed in duplicate wells. Data were analyzed by using FCAP Array 0.1 and BD Cytometric Bead Array 1.4 software assay. The theoretical limits of detection were listed as follows: IL- $1 \beta(2.3 \mathrm{pg} / \mathrm{ml})$, IL-6 $(1.6 \mathrm{pg} / \mathrm{ml})$, TNF- $\alpha(1.2 \mathrm{pg} / \mathrm{ml}), \operatorname{IFN}-\alpha(1.5 \mathrm{pg} / \mathrm{ml}), \operatorname{IFN}-\gamma(1.8 \mathrm{pg} / \mathrm{ml})$, IP-10 (0.5 pg/ml), IL-8 (1.2 pg/ml), RANTES (0.002 pg/ml), MCP-1 (1.3 pg/ml), MIG (1.1 pg/ml).

\section{Statistical analysis}

Statistical significance was determined by Two-Way ANOVA or the Mann-Whitney rank sum tests. All analyses were performed using the Statistical Package for 
Social Sciences (SPSS13.0) software (SPSS Inc, IL, USA). A probability $P<0.05$ was considered statistically significant.

\section{Abbreviations}

(EV71): Enter virus; (TLRs): 71Toll-like receptors; (RIG-I): Retinoic acid-inducible gene I; (RLHs): RIG-I-like helicases; (MDMs): Monocyte-derived macrophages; (MDA5): Melamoma differentiation associated gene 5; (HFMD):

Hand-foot-and-mouth disease; (BE): Brain stem encephalitis; (PE):

Pulmonary edema; (CNS): Central nervous system; (BBB): Blood brain barrier: (MOI): Multiplicity of infection; (CSF): Cerebrospinal fluid; (PBMCs):

Peripheral blood mononuclear cells.

\section{Competing interests}

The authors declare that they have no competing interests.

\section{Authors' contributions}

Conceived and designed the experiments: ZD, YJ, XG and JZ. Performed the experiments: XG and JZ. Analyzed the data: XG and JZ. Contributed reagents/materials/analysis tools: $W Z, N L, J L$ and $L L$. Wrote the paper: $X G, J Z, W Z$ and ZD. All authors read and approved the final manuscript.

\section{Acknowledgements}

This work was supported by China Mega-Project for Infectious Disease (2011ZX10004-001) and the National Basic Research Program (973) of China (2010CB534004)

\section{Author details}

'School of Basic Medical Sciences, Lanzhou University, Lanzhou 730000, PR China. ${ }^{2}$ National Institute for Viral Disease Control and Prevention, China CDC, Beijing 100052, PR China. ${ }^{3}$ Nanjingn Children's Hospital, Medical School of Nanjing University, Nanjing 210093, PR China.

Received: 13 January 2012 Accepted: 31 August 2012

Published: 21 September 2012

\section{References}

1. Weng KF, Chen LL, Huang PN, Shih SR: Neural pathogenesis of enterovirus 71 infection. Microbes Infect 2010, 12(7):505-510.

2. Lum LC, Wong KT, Lam SK, Chua KB, Goh AY, Lim WL, Ong BB, Paul G, AbuBakar S, Lambert M: Fatal enterovirus 71 encephalomyelitis. J Pediatr 1998, 133(6):795-798.

3. Ho M, Chen ER, Hsu KH, Twu SJ, Chen KT, Tsai SF, Wang JR, Shih SR: An epidemic of enterovirus 71 infection in Taiwan. Taiwan Enterovirus Epidemic Working Group. N Engl J Med 1999, 341(13):929-935.

4. Kim KH: Enterovirus 71 infection: an experience in Korea, 2009. Korean J Pediatr 2010, 53(5):616-622.

5. Yang F, Ren L, Xiong Z, Li J, Xiao Y, Zhao R, He Y, Bu G, Zhou S, Wang J, et al: Enterovirus 71 outbreak in the People's Republic of China in 2008.J Clin Microbiol 2009, 47(7):2351-2352.

6. Singh S, Chow VT, Phoon MC, Chan KP, Poh CL: Direct detection of enterovirus 71 (EV71) in clinical specimens from a hand, foot, and mouth disease outbreak in Singapore by reverse transcription-PCR with universal enterovirus and EV71-specific primers. J Clin Microbiol 2002, 40(8):2823-2827.

7. Shimizu H, Utama A, Yoshii K, Yoshida H, Yoneyama T, Sinniah M, Yusof MA, Okuno Y, Okabe N, Shih SR, et al: Enterovirus 71 from fatal and nonfatal cases of hand, foot and mouth disease epidemics in Malaysia, Japan and Taiwan in 1997-1998. Jpn J Infect Dis 1999, 52(1):12-15.

8. Shih SR, Ho MS, Lin KH, Wu SL, Chen YT, Wu CN, Lin TY, Chang LY, Tsao KC, Ning $\mathrm{HC}$, et al: Genetic analysis of enterovirus 71 isolated from fatal and non-fatal cases of hand, foot and mouth disease during an epidemic in Taiwan, 1998. Virus Res 2000, 68(2):127-136

9. Lin TY, Hsia SH, Huang YC, Wu CT, Chang LY: Proinflammatory cytokine reactions in enterovirus 71 infections of the central nervous system. Clin Infect Dis 2003, 36(3):269-274

10. Wang SM, Lei HY, Su LY, Wu JM, Yu CK, Wang JR, Liu CC: Cerebrospinal fluid cytokines in enterovirus 71 brain stem encephalitis and echovirus meningitis infections of varying severity. Clin Microbiol Infect 2007, 13(7):677-682.

11. Wang SM, Lei HY, Yu CK, Wang JR, Su IJ, Liu CC: Acute chemokine response in the blood and cerebrospinal fluid of children with enterovirus 71-associated brainstem encephalitis. J Infect Dis 2008, 198(7):1002-1006.

12. Williams K, Bar-Or A, Ulvestad E, Olivier A, Antel JP, Yong WW: Biology of adult human microglia in culture: comparisons with peripheral blood monocytes and astrocytes. J Neuropathol Exp Neurol 1992, 51(5):538-549

13. Chen LC, Shyu HW, Chen SH, Lei HY, Yu CK, Yeh TM: Enterovirus 71 infection induces Fas ligand expression and apoptosis of Jurkat cells. J Med Virol 2006, 78(6):780-786.

14. Lin YW, Wang SW, Tung YY, Chen SH: Enterovirus 71 infection of human dendritic cells. Exp Biol Med (Maywood) 2009, 234(10):1166-1173.

15. Wang SM, Chen IC, Su LY, Huang KJ, Lei HY, Liu CC: Enterovirus 71 infection of monocytes with antibody-dependent enhancement. Clin Vaccine Immunol 2010, 17(10):1517-1523.

16. Triantafilou K, Orthopoulos G, Vakakis E, Ahmed MA, Golenbock DT, Lepper PM, Triantafilou M: Human cardiac inflammatory responses triggered by Coxsackie B viruses are mainly Toll-like receptor (TLR) 8-dependent. Cell Microbiol 2005, 7(8):1117-1126.

17. Triantafilou K, Vakakis E, Orthopoulos G, Ahmed MA, Schumann C, Lepper PM, Triantafilou M: TLR8 and TLR7 are involved in the host's immune response to human parechovirus 1. Eur J Immunol 2005, 35(8):2416-2423.

18. Triantafilou K, Vakakis E, Richer EA, Evans GL, Villiers JP, Triantafilou M: Human rhinovirus recognition in non-immune cells is mediated by Toll-like receptors and MDA-5, which trigger a synergetic proinflammatory immune response. Virulence 2011, 2(1):22-29.

19. Xu J, Yang Y, Wang C, Jiang B: Rotavirus and coxsackievirus infection activated different profiles of toll-like receptors and chemokines in intestinal epithelial cells. Inflamm Res 2009, 58(9):585-592.

20. Brown WF: Variance estimation in the reed-muench fifty per cent end-point determination. Am J Hyg 1964, 79:37-46.

21. Strizki JM, Albright AV, Sheng H, O'Connor M, Perrin L, Gonzalez-Scarano F: Infection of primary human microglia and monocyte-derived macrophages with human immunodeficiency virus type 1 isolates: evidence of differential tropism. J Virol 1996, 70(11):7654-7662.

22. Wahid R, Cannon MJ, Chow M: Dendritic cells and macrophages are productively infected by poliovirus. J Virol 2005, 79(1):401-409.

23. Zhou J, Law HK, Cheung CY, Ng IH, Peiris JS, Lau YL: Differential expression of chemokines and their receptors in adult and neonatal macrophages infected with human or avian influenza viruses. J Infect Dis 2006, 194(1):61-70.

24. Lin TY, Chang LY, Huang YC, Hsu KH, Chiu CH, Yang KD: Different proinflammatory reactions in fatal and non-fatal enterovirus 71 infections: implications for early recognition and therapy. Acta Paediatr 2002, 91(6):632-635.

25. Wang SM, Lei HY, Huang KJ, Wu JM, Wang JR, Yu CK, Su IJ, Liu CC: Pathogenesis of enterovirus 71 brainstem encephalitis in pediatric patients: roles of cytokines and cellular immune activation in patients with pulmonary edema. J Infect Dis 2003, 188(4):564-570.

26. Husseini $\mathrm{RH}$, Sweet $\mathrm{C}$, Collie $\mathrm{MH}$, Smith $\mathrm{H}$ : Elevation of nasal viral levels by suppression of fever in ferrets infected with influenza viruses of differing virulence. J Infect Dis 1982, 145(4):520-524.

27. Chen CS, Yao YC, Lin SC, Lee YP, Wang YF, Wang JR, Liu CC, Lei HY, Yu CK: Retrograde axonal transport: a major transmission route of enterovirus 71 in mice. J Virol 2007, 81(17):8996-9003.

28. Fiala M, Looney DJ, Stins M, Way DD, Zhang L, Gan X, Chiappelli F, Schweitzer ES, Shapshak P, Weinand M, et al: TNF-alpha opens a paracellular route for HIV-1 invasion across the blood-brain barrier. Mol Med 1997, 3(8):553-564.

29. Kimura Y: Cytokines and chemokines induced by influenza virus infection. Nihon Rinsho 2006, 64(10):1822-1827.

30. Lei $X$, Liu $X, M a Y$, Sun $Z$, Yang $Y$, Jin $Q$, He B, Wang J: The $3 C$ protein of enterovirus 71 inhibits retinoid acid-inducible gene I-mediated interferon regulatory factor 3 activation and type I interferon responses. J Virol 2010, 84(16):8051-8061.

31. Ostergaard C, Benfield TL, Sellebjerg F, Kronborg G, Lohse N, Lundgren JD: Interleukin-8 in cerebrospinal fluid from patients with septic and aseptic meningitis. Eur J Clin Microbiol Infect Dis 1996, 15(2):166-169.

32. Kawai T, Akira S: Innate immune recognition of viral infection. Nat Immunol 2006, 7(2):131-137. 
33. Shalekoff S, Gray GE, Tiemessen CT: Age-related changes in expression of CXCR4 and CCR5 on peripheral blood leukocytes from uninfected infants born to human immunodeficiency virus type 1-infected mothers. Clin Diagn Lab Immunol 2004, 11(1):229-234.

34. Hamaguchi T, Fujisawa H, Sakai K, Okino S, Kurosaki N, Nishimura Y, Shimizu $H$, Yamada M: Acute encephalitis caused by intrafamilial transmission of enterovirus 71 in adult. Emerg Infect Dis 2008, 14(5):828-830.

doi:10.1186/1471-2334-12-224

Cite this article as: Gong et al:: Excessive proinflammatory cytokine and

chemokine responses of human monocyte-derived macrophages to

enterovirus 71 infection. BMC Infectious Diseases 2012 12:224.

\section{Submit your next manuscript to BioMed Central and take full advantage of:}

- Convenient online submission

- Thorough peer review

- No space constraints or color figure charges

- Immediate publication on acceptance

- Inclusion in PubMed, CAS, Scopus and Google Scholar

- Research which is freely available for redistribution 